\title{
BMJ Open Better outcome from arthroscopic partial meniscectomy than skin incisions only? A sham-controlled randomised trial in patients aged 35-55 years with knee pain and an MRI-verified meniscal tear
}

\author{
Ewa M Roos, ${ }^{1}$ Kristoffer Borbjerg Hare, ${ }^{1,2}$ Sabrina Mai Nielsen, ${ }^{3}$ \\ Robin Christensen, ${ }^{3}$ L Stefan Lohmander ${ }^{4}$
}

To cite: Roos EM, Hare KB, Nielsen SM, et al. Better outcome from arthroscopic partial meniscectomy than skin incisions only? A shamcontrolled randomised trial in patients aged $35-55$ years with knee pain and an MRI-verified meniscal tear. BMJ Open 2018;8:e019461. doi:10.1136/ bmjopen-2017-019461

- Prepublication history and additional material for this paper are available online. To view these files, please visit the journal online (http://dx.doi org/10.1136/bmjopen-2017019461).

Received 7 September 2017 Revised 19 December 2017 Accepted 22 December 2017

\section{Check for updates}

${ }^{1}$ Department of Sport and Clinical Biomechanics, University of Southern Denmark, Odense, Denmark

${ }^{2}$ Department of Orthopaedics, Slagelse Hospital, Slagelse, Denmark

${ }^{3}$ Musculoskeletal Statistics Unit, The Parker Institute, Bispebjerg and Frederiksberg Hospital, Copenhagen, Denmark

${ }^{4}$ Department of Clinical Sciences Lund, Orthopaedics, Lund University, Lund, Sweden

Correspondence to

Dr Ewa M Roos;

eroos@health.sdu.dk

\section{ABSTRACT}

Objective Compare arthroscopic partial meniscectomy to a true sham intervention.

Methods Sham-controlled superiority trial performed in three county hospitals in Denmark comparing arthroscopic partial meniscectomy to skin incisions only in patients aged 35-55 years with persistent knee pain and an MRIconfirmed medial meniscus lesion. A computer-generated table of random numbers generated two comparison groups. Participants and outcome assessors were blinded to group allocation. Exclusions were locking knees, highenergy trauma or severe osteoarthritis. Outcomes were collected at baseline, 3 and 24 months. We hypothesised no difference between groups. The primary outcome was the between-group difference in change from baseline to 2 years in the mean score across all five normalised Knee injury and Osteoarthritis Outcome Score (KOOS) subscales $\left(\mathrm{KOOS}_{5}\right)$.

Results Forty-four patients (of the estimated 72) underwent randomisation; 22 in each group. Sixteen participants (36\%) were non-blinded and eight participants $(36 \%)$ from the sham group crossed over to the surgery group prior to the 2-year follow-up. At 2 years, both groups reported clinically relevant improvements (surgery 21.8, skin incisions only 13.6), the mean difference between groups was 8.2 in favour of surgery, which is slightly less than the cut-off of 10 prespecified to represent a clinically relevant difference; judged by the $95 \% \mathrm{Cl}(-3.4$ to 19.8), a possibility of clinically relevant difference could not be excluded. In total, nine participants experienced 11 adverse events; six in the surgery group and three in the skin-incisions-only group.

Conclusion We found greater improvement from arthroscopic partial meniscectomy compared with skin incisions only at 2 years, with the statistical uncertainty of the between-group difference including what could be considered clinically relevant. Because of the study being underpowered, nearly half in the sham group being non-blinded and one-third crossing over to surgery, the results cannot be generalised to the greater patient population.

Trial registration number NCT01264991.
Strengths and limitations of this study

- This is only the second trial to compare arthroscopic partial meniscectomy with sham surgery intervention in patients with MRI-verified meniscus lesion.

- This trial includes younger patients than previous trials of arthroscopic surgery.

- With the study being underpowered, nearly half in the sham group being non-blinded and one-third crossing over to surgery, the results cannot be generalised to the greater patient population.

- Our trial confirms the challenges of performing sham-controlled trials of surgery.

\section{INTRODUCTION}

Knee pain is responsible for a large and increasing disease burden, including in middle-aged and older people. ${ }^{1}$ Recommended management strategies include patient education, exercise, weight loss, analgesics and surgery. ${ }^{2-4}$ Notwithstanding it being very commonly practised, systematic reviews have found no added benefit from knee arthroscopic surgery in the chronically painful knee of middle-aged or older persons. ${ }^{56}$ While there are at least seven randomised controlled trials of arthroscopic surgery in patients with knee osteoarthritis, only three have been done in patients with a meniscal tear but without established osteoarthritis. ${ }^{7-9}$ Patients included in these studies were middle-aged (35 and older) with mean ages ranging from 49 to 54 years. ${ }^{7-9}$ Two of these studies used exercise as the comparator, either head-to-head ${ }^{9}$ or in an additive design. ${ }^{7}$ Only one of these studies was able to control for non-specific (placebo) effects by comparing arthroscopic partial meniscectomy to diagnostic arthroscopy. ${ }^{8}$ However, some argue that diagnostic arthroscopy may 
have a treatment effect in itself since the joint is entered with instruments and lavage is performed. ${ }^{10}$

While the number of arthroscopic procedures, with or without meniscectomy, to treat people with established osteoarthritis may have decreased over the last decade, ${ }^{1112}$ the number of arthroscopic partial meniscectomies has risen. ${ }^{11}$ One reason for the continued high use of knee arthroscopy may be the few sham knee arthroscopy trials, ${ }^{8} 13$ with only one for arthroscopic partial meniscectomy. ${ }^{8}$ The non-specific effects of surgery and other invasive procedures are generally large. ${ }^{14}$ Particularly in the field of pain-related conditions, more evidence from randomised placebo-controlled trials is required to avoid continuation of ineffective treatments. ${ }^{15} 16$ To compare arthroscopic partial meniscectomy to a true sham intervention, we performed a randomised controlled trial comparing arthroscopic partial meniscectomy to skin incisions only in middle-aged patients with persistent knee pain and an MRI-verified meniscus lesion.

\section{METHODS}

\section{Trial design}

We conducted this blinded randomised sham-controlled multicentre trial at three county hospitals in Denmark from February 2011 to March 2015. Details of the trial design and methods are published. ${ }^{17}$ The study was consistent with the Declaration of Helsinki. The first and second authors vouch for the accuracy and completeness of the reported data, analyses and adherence to the previously published registration (ClinicalTrials.gov number NCT01264991), protocol $^{17}$ and the corresponding final statistical analysis plan (online supplementary appendix). All patients provided written informed consent. On entering the study, patients were informed that if they were randomised to sham treatment and did not experience satisfactory pain relief, they could cross over to the other procedure (arthroscopic partial meniscectomy) by 3 months or later.

\section{Participants}

According to the protocol, eligible patients were between 35 and 55 years of age with knee pain for more than 2 months and an MRI-confirmed medial meniscus lesion, but without significant trauma. Patients were excluded if they had experienced prolonged episodes of inability to fully extend the knee, having had high-energy trauma, grade 3-4 osteoarthritis on the Kellgren and Lawrence classification or knee surgery within the previous 2 years. Detailed inclusion and exclusion criteria are provided in online supplementary appendix table 1.

\section{Blinding and randomisation}

All study personnel and participants were blinded to the intervention, except for the surgeons and other operating theatre personnel, who did not have any other role in the study. Two comparison groups were generated by an external coinvestigator using simple randomisation with an equal allocation ratio (1:1), by referring to a computer-generated table of random numbers. Block randomisation with blocks of four or six was used, and patients were stratified for treatment centre. Once the patient was sedated, the surgeon retrieved a sealed consecutively numbered envelope containing the patient's assigned intervention from a briefcase kept just outside the actual operating theatre.

\section{Protocol deviations}

When registering the trial, we were concerned about dropouts and therefore aimed to recruit 100 patients despite only 72 being needed to obtain a power of $80 \% .^{17}$ During the trial recruitment period, several relevant studies on knee arthroscopy were published and received substantial media attention in Denmark. This greatly impacted the ability to recruit patients to the study. As a consequence, we arranged for a third centre to help with recruitment. Despite large efforts, this centre was able to recruit only two patients for the study. Therefore, for feasibility and ethical reasons, we closed the recruitment on 29 January 2015 after a recruitment period of 51 months, and report the results for the 44 patients recruited, enrolled and randomised. Considering the nature and rarity of a sham-controlled surgical trial, we considered it important and ethically prudent to make the results publicly available. Minor protocol deviations were that the Knee injury and Osteoarthritis Outcome Score (KOOS) questionnaire was not completed by the patients prior to crossing over to the surgery arm, and we did not play a video showing a standard arthroscopy during the sham procedure.

\section{Operative and postoperative procedures}

In both groups, all procedures were performed with general anaesthesia combined with local anaesthesia. The knee was tested for stability, and two skin incisions were made. Ten experienced surgeons in their final year of residency, or attending orthopaedic surgeons, performed the procedures.

In the arthroscopic partial meniscectomy group, a tourniquet was used at the discretion of the surgeon. Normal procedures were followed, and the arthroscope was inserted. The strategy for the meniscectomy was to preserve as much tissue as possible. No other surgical procedures were performed. A standard protocol was used to document perioperative findings in cartilage and the medial and lateral menisci.

In the skin-incisions-only group, following the initial common procedures, the knee was manipulated as if real arthroscopy was performed; the spillage of water and all other equipment needed for an arthroscopy was used. No instruments were entered into the incisions to minimise the risk of a deep infection, osteochondral lesion or unwanted intervention by the surgeon.

In both groups, patients were handed the same standard care folder including postoperative advice and a home-based exercise programme. At 1 week, biking, 
swimming and fast walking were recommended, and at 2-3 weeks, more intense biking and jogging were recommended. For the first postoperative week, seven different non-weight-bearing exercises to improve lower extremity function and knee range of motion were suggested, and an additional three weight bearing thereafter. All exercises were recommended to be performed 10-15 times three times daily (online supplementary appendix).

\section{Outcome measures}

All outcomes were collected at baseline, and at 3 and 24 months' follow-ups. The prespecified primary outcome measure was the between-group difference in change from baseline to 2 years in the mean score across all five normalised KOOS subscales, covering pain, symptoms, activities of daily living, sport and recreation function, and quality of life $\left(\operatorname{KOOS}_{5}\right)$. Each subscale consists of multiple items scored on a 5-point Likert scale; the $\mathrm{KOOS}_{5}$ ranges from 0 to 100, worst to best. KOOS is a valid, reliable and responsive measure of patient-reported outcomes for patients having knee arthroscopy. ${ }^{18} 19$ To minimise bias, questionnaires were filled out at home at all time points.

Secondary patient-reported outcome measures were the five individual KOOS subscales, the Short-Form 36-item (SF-36) Physical and Mental Component Scores, a seven-step Global Perceived Effect (GPE) score (ranging from much worse to much better) and the EuroQolGroup 5-dimension 3-level (EQ-5D-3L). Three objective measures of lower extremity function were collected, with elastic stockings concealing both knees and randomisation of first leg to be tested at each visit to minimise bias. The best value for each test was recorded, and the details of the testing procedures were described. ${ }^{17}$ Maximum knee extension force was recorded as maximal voluntary torque per $\mathrm{kg}$ of body mass and measured sitting using a hand-held dynamometer. The one-leg hop test, measured in centimetres, is a measure of function at a level above daily living and recommended for middle-aged subjects at risk of osteoarthritis. ${ }^{20}$ The maximum number of one-legged knee-bends in $30 \mathrm{~s}$ resembles stepping down a stair and was included as a measure of one-legged function at the level of daily living valid and reliable in patients who had undergone meniscectomy. ${ }^{21}$

\section{Statistical analysis}

The primary analysis of the primary outcome measure $\left(\mathrm{KOOS}_{5}\right)$ was conducted according to the intention-totreat (ITT) principle; the ITT principle implies that all randomised patients should be analysed according to the treatment to which the patient was allocated, irrespective of whether they received this or some other treatment, or no treatment at all.

The statistical analyses were based on a repeated linear mixed model including all patients with a baseline assessment, with both fixed (group, centre and time) and random (patient identity) factors. We prespecified the primary analyses in this trial to be based on the 'as observed cases' while respecting the randomisation (ie, no data imputation). ${ }^{22}$ To explore the robustness of these primary analyses, the Baseline Observation Carried Forward (ie, null responder) imputation techniques, as well as the 'Per Protocol' population, was also applied for the purpose of sensitivity analysis.

\section{RESULTS}

\section{Characteristics of the patients}

Of the 235 patients who were eligible for enrolment prior to having an MRI scan, 135 declined to participate (figure 1). Of the 100 willing to participate, 24 did not have a meniscal lesion on MRI, and 32 declined after a meniscal lesion was confirmed on MRI. Thus, a total of 44 patients were enrolled and underwent randomisation; 22 were randomised to arthroscopic partial meniscectomy and 22 to skin incisions only (sham procedure). The baseline characteristics of the two groups were well balanced (table 1). All underwent the assigned intervention.

\section{Missing data}

Due to an administrative error, two sets of questionnaires (from the skin-incisions group) were missing at baseline. At 2 years, two (other) participants were lost to follow-up, both from the skin-incisions group (these two subjects crossed over to arthroscopic surgery between 3 and 24 months), yielding a follow-up rate of $96 \%$. Functional testing was not performed in 3 patients at baseline and in 10 patients at the 2-year follow-up (figure 1). The most common reason given was long travel distance to the test centre, however notably 8 out of 10 participants not tested at follow-up were previously non-blinded.

\section{Non-blinding and cross-over to arthroscopic partial meniscectomy}

In total, 16 participants $(36 \% ; 6$ from the arthroscopy group and 10 from the sham group) were non-blinded prior to the 2-year follow-up. One patient from the skin-incisions group was non-blinded by a nurse directly following surgery. Prior to 3 months, two additional patients were non-blinded by the treating surgeon because of suspicion of an adverse event (one infection and one thrombosis, neither later confirmed, both from the arthroscopy group) and one because of persisting pain (from the skin-incisions group). Between 3 and 24 months, two from the arthroscopy group and eight from the sham group were non-blinded by the treating surgeon because of persisting pain. One from the arthroscopy group was informed about group allocation by his general practitioner (GP) checking the digital medical records, and one participant accessed the information himself in his digital medical records.

Arthroscopic partial meniscectomy was performed in 8 out of 10 non-blinded cases in the skin-incisions group, yielding a cross-over rate of $36 \%$. 


\section{Primary outcome, ITT population}

At 3 months, both groups reported clinically important and statistically significant improvements of 13.4 and 14.9 points in $\mathrm{KOOS}_{5}$ favouring the skin-incisions group only, with no significant difference between groups $(1.5,95 \%$ CI: -10.0 to $13.0, \mathrm{P}=0.796)$. At 2 years, the primary endpoint of this trial, the group randomised to arthroscopic partial meniscectomy continued to improve and reported a clinically relevant improvement of 21.8

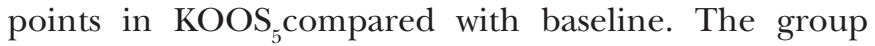
randomised to skin incisions only did not improve further from 3 months; the average improvement from baseline was however still clinically relevant, 13.6 points. The mean difference between groups at 2 years was 8.2 , which is slightly less than the cut-off of 10 prespecified to represent a clinically relevant difference (table 2 and figure 2 ). The 95\% CI (-3.4 to 19.8) included the predefined cut-off for a clinically relevant difference (table 2 and figure 3 ).

\section{Secondary and other outcomes}

The individual KOOS subscales at 2years largely supported the primary outcome with between-group differences of 11.5 for quality of life (QOL), 9.9 for pain, 9.0 for ADL, 6.6 for symptoms and 5.6 for sport/recreation, all in favour of arthroscopic partial meniscectomy. The mean differences for QOL and pain were 10 or

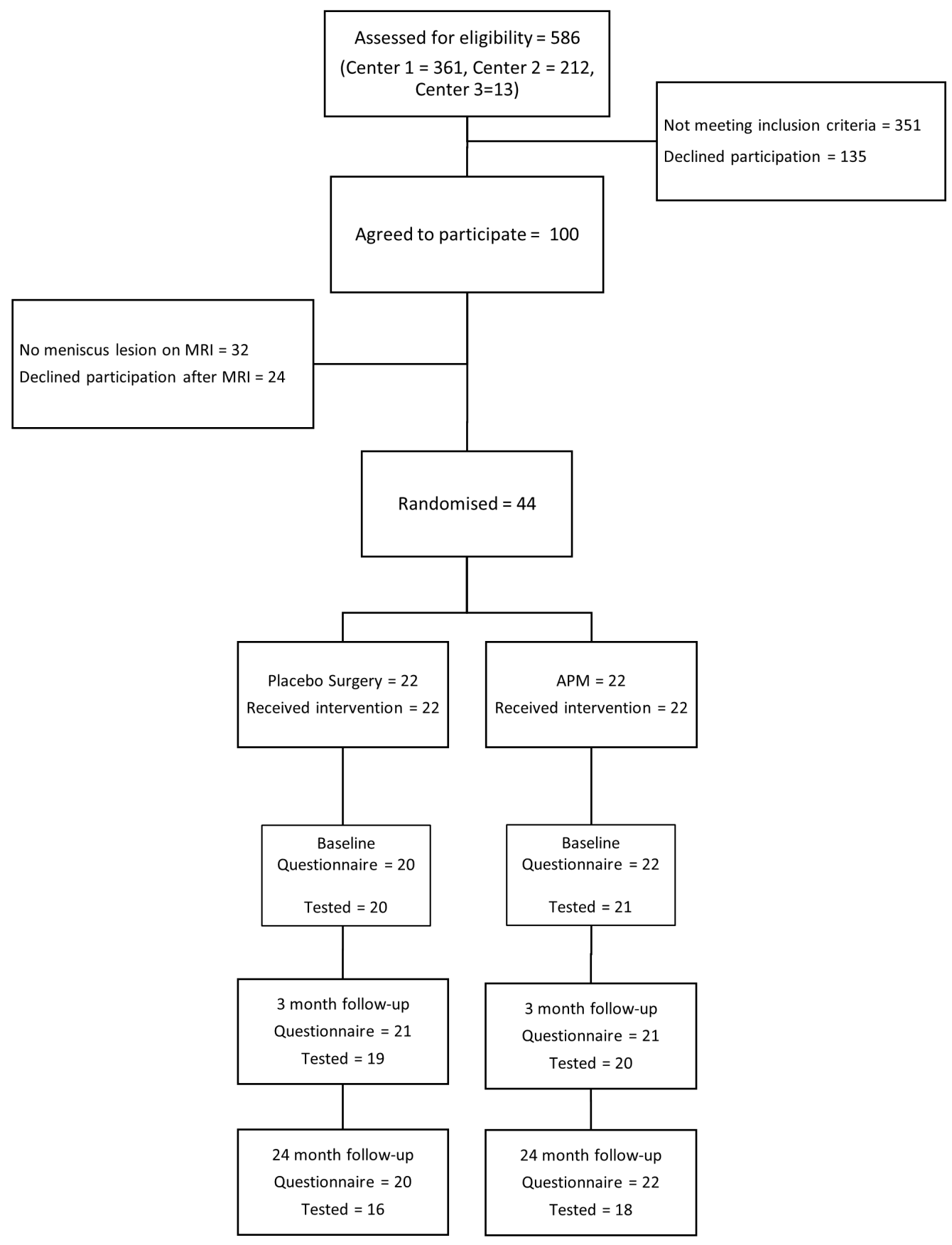

Figure 1 Study flow. 
Table 1 Patient characteristics

\begin{tabular}{|c|c|c|c|c|c|c|c|c|}
\hline \multirow[b]{2}{*}{ Variable } & \multirow[b]{2}{*}{$\mathbf{N}$} & \multirow{2}{*}{$\begin{array}{l}\text { Arthroscopic } \\
\text { partial } \\
\text { meniscectomy }\end{array}$} & \multirow[b]{2}{*}{$\mathbf{N}$} & \multirow{2}{*}{$\begin{array}{l}\text { Skin incisions } \\
\text { only } \\
\text { Mean (SD) }\end{array}$} & \multirow[b]{2}{*}{$\mathbf{N}$} & \multirow{2}{*}{$\begin{array}{l}\text { Total } \\
\text { Mean (SD) }\end{array}$} & \multirow[b]{2}{*}{ Min } & \multirow[b]{2}{*}{ Max } \\
\hline & & & & & & & & \\
\hline \multicolumn{9}{|l|}{ Person demographics } \\
\hline Age, years & 22 & $47.2(5.9)$ & 22 & $46.4(5.5)$ & 44 & $46.8(5.7)$ & 37.5 & 55.8 \\
\hline Females, n (\%) & 22 & $9(41)$ & 22 & $12(55)$ & 44 & $21(48)$ & & \\
\hline Weight, kg & 22 & $85.6(14.6)$ & 22 & $79.7(19.5)$ & 44 & $82.6(17.3)$ & 52 & 120 \\
\hline $\mathrm{BMI}, \mathrm{kg} / \mathrm{m}^{2}$ & 22 & $27.6(3.6)$ & 22 & $26(3.9)$ & 44 & $26.8(3.8)$ & 18.6 & 33.5 \\
\hline \multicolumn{9}{|l|}{ Knee examinations } \\
\hline Kellgren and Lawrence grade $(0-4)$ & 22 & & 22 & & 44 & & & \\
\hline $0, \mathrm{n}(\%)$ & & $9(41)$ & & $10(45)$ & & $19(43)$ & & \\
\hline 4, n (\%) & & $0(0)$ & & $0(0)$ & & $0(0)$ & & \\
\hline Joint line tenderness, n (\%) & 21 & $21(100)$ & 22 & $20(91)$ & 43 & $41(95)$ & & \\
\hline Positive results of McMurray test, $\mathrm{n}(\%)$ & 21 & $17(81)$ & 22 & $17(77)$ & 43 & $34(79)$ & & \\
\hline Swelling present, n (\%) & 21 & $11(52)$ & 22 & $8(36)$ & 43 & $19(44)$ & & \\
\hline Knee extension & 21 & & 22 & & 43 & & & \\
\hline Full extension, $\mathrm{n}(\%)$ & & $18(86)$ & & $21(95)$ & & $39(91)$ & & \\
\hline Small extension deficit $<10^{\circ}, \mathrm{n}(\%)$ & & $3(14)$ & & $1(5)$ & & $4(9)$ & & \\
\hline Knee flexion & 21 & & 22 & & 43 & & & \\
\hline Full flexion, n (\%) & & $17(81)$ & & $14(64)$ & & $31(72)$ & & \\
\hline \multicolumn{9}{|l|}{ Findings during arthroscopy } \\
\hline $\begin{array}{l}\text { Extent of resection ( } 1-3 \text { regions), median } \\
\text { (range) }\end{array}$ & & $1(1-3)$ & & & & & & \\
\hline Anterior region, $\mathrm{n}(\%)$ & & $1(5)$ & & & & & & \\
\hline Mid region, n (\%) & & $1(5)$ & & & & & & \\
\hline Posterior region, n (\%) & & $19(90)$ & & & & & & \\
\hline \multicolumn{9}{|l|}{ Cartilage status: (worst ICRS grade, 0-IVb) } \\
\hline Tibia, median (range) & & $0(0-I I I b)$ & & & & & & \\
\hline Femur, median (range) & & 0.5 (0- IIIa) & & & & & & \\
\hline Patella, median (range) & & $0(0-I I)$ & & & & & & \\
\hline \multicolumn{9}{|l|}{ Patient-reported data } \\
\hline Duration of pain, months, median (IQR) & 22 & $5.0(2.0-6.0)$ & 22 & $3.5(2.0-6.0)$ & 44 & $4.0(2.0-6.0)$ & 2 & 60 \\
\hline \multicolumn{9}{|l|}{ KOOS } \\
\hline $\mathrm{KOOS}_{5}(0-100)$ & 22 & $51.2(15.6)$ & 20 & $44.8(19.9)$ & 42 & $48.1(17.8)$ & 21 & 88 \\
\hline Pain $(0-100)$ & 22 & $55.1(15.4)$ & 20 & $45.9(22)$ & 42 & $50.7(19.2)$ & 11 & 86 \\
\hline Symptoms (0-100) & 22 & $62.8(17.7)$ & 20 & $59.9(20.6)$ & 42 & $61.4(18.9)$ & 32 & 100 \\
\hline Function in daily living $(0-100)$ & 22 & $64.9(19.9)$ & 20 & $56.5(22.3)$ & 42 & $60.9(21.3)$ & 25 & 100 \\
\hline Function in sport and recreation (0-100) & 22 & $35(23)$ & 20 & $25.2(26.3)$ & 42 & $30.4(24.9)$ & 0 & 85 \\
\hline Knee-related quality of life $(0-100)$ & 22 & $38.7(15.4)$ & 20 & $36.6(20.2)$ & 42 & $37.7(17.6)$ & 6 & 75 \\
\hline
\end{tabular}


Table 1 Continued

\begin{tabular}{|c|c|c|c|c|c|c|c|c|}
\hline \multirow[b]{2}{*}{ Variable } & \multirow[b]{2}{*}{$\mathbf{N}$} & \multirow{2}{*}{$\begin{array}{l}\begin{array}{l}\text { Arthroscopic } \\
\text { partial } \\
\text { meniscectomy }\end{array} \\
\text { Mean (SD) }\end{array}$} & \multirow[b]{2}{*}{$\mathbf{N}$} & \multirow{2}{*}{$\begin{array}{l}\text { Skin incisions } \\
\text { only } \\
\text { Mean (SD) }\end{array}$} & \multirow[b]{2}{*}{$\mathbf{N}$} & \multirow{2}{*}{$\begin{array}{l}\text { Total } \\
\text { Mean (SD) }\end{array}$} & \multirow[b]{2}{*}{ Min } & \multirow[b]{2}{*}{ Max } \\
\hline & & & & & & & & \\
\hline \multicolumn{9}{|l|}{$E Q-5 D$} \\
\hline VAS score & 21 & $69(14)$ & 20 & 63 & 41 & $66(19)$ & 25 & 95 \\
\hline EQ-5D-3L index value $(0-1)$ & 21 & $0.749(0.108)$ & 20 & 0.642 & 41 & $0.697(0.148)$ & 0.293 & 1.000 \\
\hline Physical component summary $(0-100)$ & 21 & $38(10)$ & 20 & 35 & 41 & $37(9)$ & 17 & 55 \\
\hline Mental component summary $(0-100)$ & 21 & $59(7)$ & 20 & 57 & 41 & $58(7)$ & 43 & 70 \\
\hline \multicolumn{9}{|l|}{ Performance and muscle function } \\
\hline One leg hop test (affected knee), cm & 21 & $54(33)$ & 20 & 52 & 41 & $53(33)$ & 0 & 130 \\
\hline One leg hop test (non-affected knee), cm & 21 & $64(30)$ & 20 & 64 & 41 & $64(30)$ & 4 & 126 \\
\hline $\begin{array}{l}\text { Knee bending test, max number of knee } \\
\text { bends (non-affected knee), } n\end{array}$ & 21 & $21(7)$ & 20 & 19 & 41 & $20(7)$ & 8 & 33 \\
\hline Knee bendings LSI*, \% & & 86 & & 79 & & 85 & & \\
\hline $\begin{array}{l}\text { Isometric knee extensor strength (affected } \\
\text { knee), } \mathrm{Nm} / \mathrm{kg}\end{array}$ & 21 & $0.96(0.59)$ & 20 & 0.78 & 41 & $0.87(0.55)$ & 0.00 & 2.06 \\
\hline $\begin{array}{l}\text { Isometric knee extensor strength (non- } \\
\text { affected knee), } \mathrm{Nm} / \mathrm{kg}\end{array}$ & 21 & $1.11(0.49)$ & 20 & 0.99 & 41 & $1.05(0.47)$ & 0.33 & 2.06 \\
\hline Isometric knee extensor strength LSI*, \% & & 86 & & 79 & & 83 & & \\
\hline
\end{tabular}

Data are presented as mean and SD unless otherwise stated.

* LSI is calculated as 100 (unaffected leg-affected leg).

BMI, body mass index; ICRS,International Cartilage Repair Society; KOOS, Knee injury and Osteoarthritis Outcome Score; LSI, Limb

Symmetry Index; SF-36, Short-Form 36 items; VAS, Visual Analogue Scale.

greater (depending on rounding), a suggested cut-off for being clinically relevant (figure 3 ). None of these differences were statistically significant (table 2).

There were no statistically significant between-group differences in EQ-5D, SF-36, GPE score, muscle strength or physical performance measures. In the surgery group, $67 \%$ reported a GPE response of 'better' or 'much better', compared with $37 \%$ in the skin-incisions group, $\mathrm{P}=0.059$ (table 2).

\section{Sensitivity analyses}

The sensitivity analyses at 2 years largely supported the primary analyses (figure 3, online supplementary appendix tables 2 and 3). Notably, in the per-protocol analysis, a non-significant 9.7-point greater improvement in $\mathrm{KOOS}_{5}$ score was seen at 3 months in the skin-incision group (figure 2).

\section{Adverse events}

Crossing over to arthroscopic partial meniscectomy in the skin-incisions group was not considered an adverse event. Four knee-related adverse events were recorded, of which two were regarded as serious (two rearthroscopies; one partial meniscectomy and one anterior cruciate ligament reconstruction), one cutaneous nerve lesion and one mild knee swelling), all in the surgery group. In total, seven other adverse events in five participants, two in the surgery group and three in the skin-incisions group, were recorded (chest pain, finger injury, nausea, dizziness and kidney stone) including two regarded as serious (abdominal surgery and malignant melanoma).

\section{DISCUSSION}

We included middle-aged (mean 47.2 years, range 37.5-55.8), slightly overweight (body mass index 26.8) patients with typical symptoms. Our patient sample is the youngest thus far included in a controlled randomised trial of arthroscopic surgery of meniscal tear. While they suffered from, on average, moderate knee pain and daily functional limitations (KOOS: pain 51, symptoms 61, ADL 61) and severely impaired sport and recreation function (KOOS: sport/recreation 30) with associated reduction in QOL (KOOS: QOL 38), only few had radiographic osteoarthritis $(8 \%)$. Single-leg muscle function was impaired with reductions of $15 \%-17 \%$ in the affected leg compared with the non-affected leg in knee extensor strength, hop performance and knee bends.

Comparing the two groups at 2 years, the $95 \%$ CI $(-3.4$ to 19.8) of the primary outcome $\mathrm{KOOS}_{5}$ included the prespecified clinically relevant difference of 10 , and a 

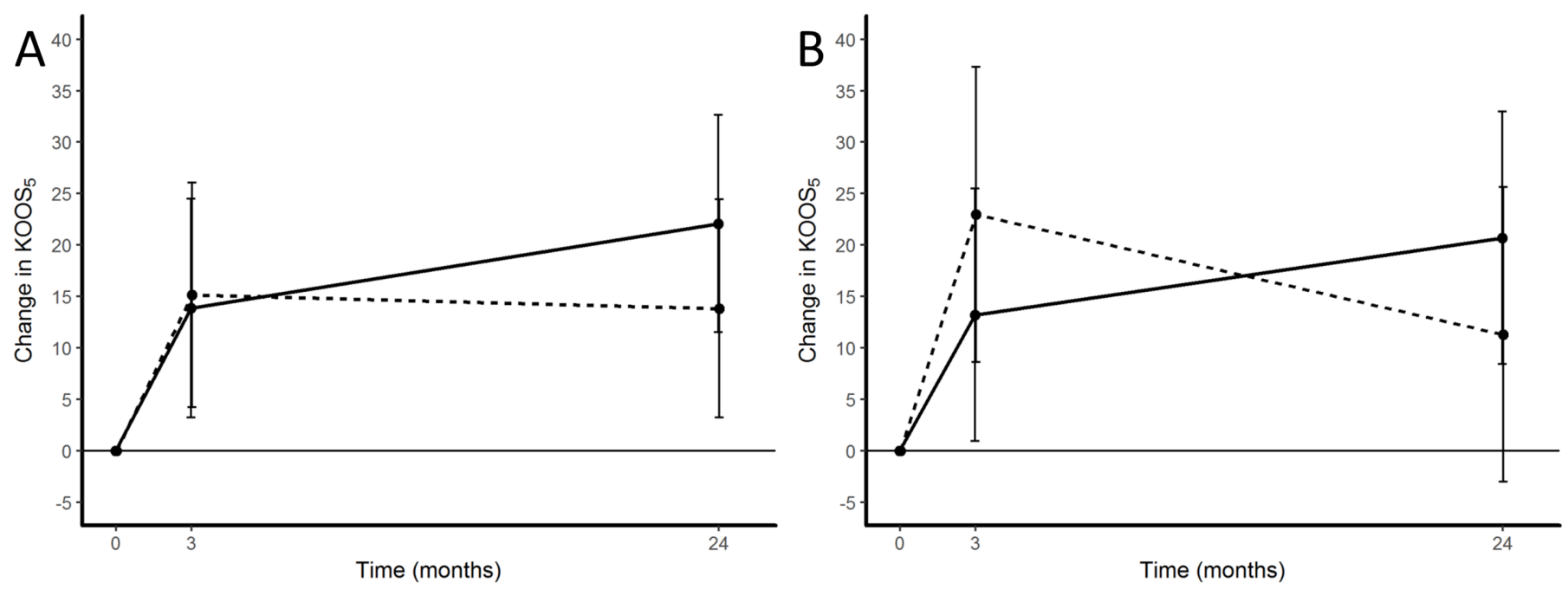

Figure 2 Least square mean for each group over time for the primary outcome $\left(\mathrm{KOOS}_{5}\right)$ for intention-to-treat $(\mathrm{A})$ and perprotocol (B) analyses. Solid lines represent the arthroscopic partial meniscectomy group and stipled lines the skin incision only group. $\mathrm{KOOS}_{5}$, the mean score across all five normalised Knee injury and Osteoarthritis Outcome Score subscales.

true difference between groups in favour of arthroscopic partial meniscectomy cannot be excluded. Secondary outcomes largely supported the primary outcome.

The results of this trial, with regard to the observed treatment effect on pain of either partial meniscectomy or sham

\section{A}

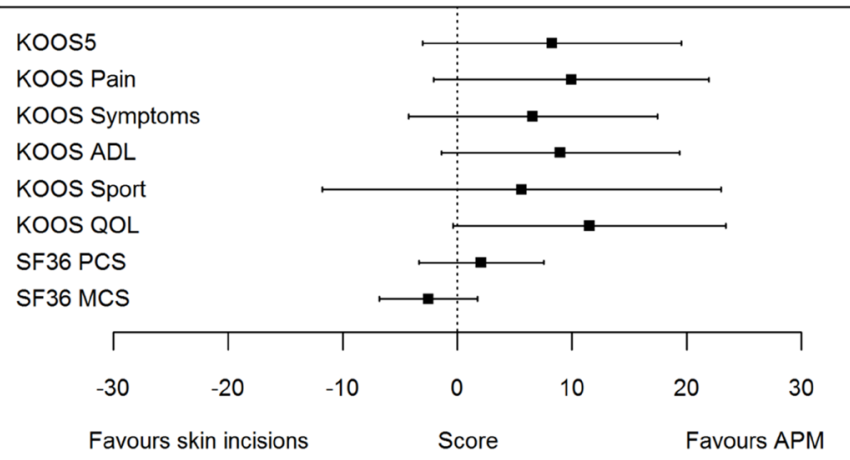

B

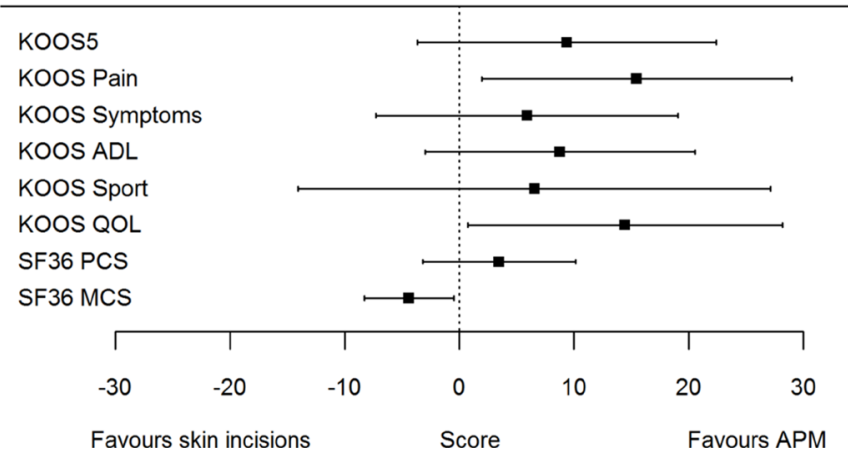

Figure 3 Forest plot illustrating the contrast between the treatment arms after 2 years for intention-to-treat $(A)$ and perprotocol (B) groups. ADL, Function, daily living; KOOS, Knee injury and Osteoarthritis Outcome Score; $\mathrm{KOOS}_{5}$, the mean score across all five normalised KOOS subscales; MCS, Mental Component Score; PCS, Physical Component Score; QOL, quality of life; SF-36. Short-Form 36 items. surgery, are well within the envelope of previous trials of arthroscopic surgery for painful knee. ${ }^{56}$

The use of a sham surgery comparator increased the rigour of our trial at the 3-month follow-up but not necessarily at the primary end point at 2 years because a high proportion of patients were non-blinded between 3 and 24 months. At 3 months in the skin-incisions group, $48 \%$ thought they received the real treatment and $70 \%$ were certain the treatment would help them. In the surgery group, $70 \%$ thought they received the real treatment and $50 \%$ believed the treatment would help them. While four patients (two from the skin-incision group) were non-blinded prior to 3 months, an additional 10 (eight from the skin-incisions group) were non-blinded between 3 and 24 months.

This study had several major limitations that highlight the well-known challenges to perform randomised surgery trials controlled with sham surgery. ${ }^{23}$ First, we managed to include only $60 \%$ of the estimated number of patients needed, resulting in low statistical power. Second, 36\% $(16 / 44)$ of patients were non-blinded in the course of the study, which may have enhanced the placebo effects in the surgery group and negatively impacted the patient-reported outcomes in the sham-surgery group through nocebo effects. ${ }^{24}$ In our trial, $45 \%$ (10/22) of patients randomised to skin incisions only were non-blinded. Third, 8 of the 10 non-blinded patients in the skin-incision-only group crossedover to meniscus resection, which further confounded the interpretation of the results. Taken together, these limitations markedly hampered our ability to generalise these results to the greater patient population. If we had managed to recruit the originally planned number of patients, fewer patients would have been non-blinded and fewer patients would have crossed over, a more firm interpretation of the results would have been possible.

Future trialists may consider increasing the number of recruiting centres in multicentre studies and providing extensive training in research methodology not only for 
those closely involved in the study but for all personnel in the operating theatre to avoid early non-blinding. Practising consultation scenarios and improved management of patients with persistent pain during follow-up visits may help avoid the cross-overs often seen in those being non-blinded before or after 3 months by the treating surgeon. In Denmark, patients and their GPs have easy digital access to patient records, including perioperative reports, and are thus able to reveal group allocation without contacting the study personnel. This was the case for two patients in our study where non-blinding could not be avoided.

\section{CONCLUSION}

We found greater improvement from arthroscopic partial meniscectomy compared with skin incisions only at 2 years, with uncertainty around the between-group difference depending on what is considered a clinically relevant difference between groups. Because of the study being underpowered, nearly half in the sham group being non-blinded and one-third crossing over to surgery, the results cannot be generalised to the greater patient population.

Acknowledgements We would like to thank the recruiting and operating surgeons Anders Kunov, Jawaid Ahmed, Karl Rønne, Kurt Skovgaard, Michael Thøger Langergaard, Peter Weis Jessen, Sten Rasmussen, Søren Bondo and Thomas Houe and the physiotherapists Christina Skou, Janne Mølgaard and Tejs Brygger Petersen involved in testing of physical performance.

Contributors EMR contributed to designing the study, the statistical analysis plan, interpreting the data and drafted the first manuscript. KBH contributed to designing the study, was responsible for patient recruitment and data collection, contributed to the final statistical analysis plan and approved the manuscript. SMN contributed to the final statistical analysis plan and analysing/interpreting the data. RC contributed to designing the study, the original and final statistical analysis plan and interpreting the data. LSL contributed to designing the study, the statistical analysis plan and interpreting the data. All authors provided intellectual input, revised and approved the final manuscript.

Funding Research Fund of Hospital South, Region Zealand; Region Zealand Health Scientific Research Fund; Edith and Henrik Henriksens Memorial Fund; The Danish Rheumatism Association. The Parker Institute, Bispebjerg and Frederiksberg Hospital (R. Christensen and S.M. Nielsen) is supported by a core grant from the Oak Foundation (OCAY-13-309).

Competing interests None declared.

Patient consent Obtained.

Ethics approval Research Ethics Committee of Region Zealand, Denmark.

Provenance and peer review Not commissioned; externally peer reviewed.

Data sharing statement № additional data available.

Open Access This is an Open Access article distributed in accordance with the Creative Commons Attribution Non Commercial (CC BY-NC 4.0) license, which permits others to distribute, remix, adapt, build upon this work non-commercially, and license their derivative works on different terms, provided the original work is properly cited and the use is non-commercial. See: http://creativecommons.org/ licenses/by-nc/4.0/

(C) Article author(s) (or their employer(s) unless otherwise stated in the text of the article) 2018. All rights reserved. No commercial use is permitted unless otherwise expressly granted.

\section{REFERENCES}

1. Cross M, Smith E, Hoy D, et al. The global burden of hip and knee osteoarthritis: estimates from the global burden of disease 2010 study. Ann Rheum Dis 2014;73:1323-30.
2. McAlindon TE, Bannuru RR, Sullivan MC, et al. OARSI guidelines for the non-surgical management of knee osteoarthritis. Osteoarthritis Cartilage 2014;22:363-88.

3. Hochberg MC, Altman RD, April KT, et al. American College of Rheumatology 2012 recommendations for the use of nonpharmacologic and pharmacologic therapies in osteoarthritis of the hand, hip, and knee. Arthritis Care Res 2012;64:465-74.

4. Fernandes L, Hagen KB, Bijlsma JW, et al. EULAR recommendations for the non-pharmacological core management of hip and knee osteoarthritis. Ann Rheum Dis 2013;72:1125-35.

5. Thorlund JB, Juhl CB, Roos EM, et al. Arthroscopic surgery for degenerative knee: systematic review and meta-analysis of benefits and harms. BMJ 2015;350:h2747.

6. Monk P, Garfjeld Roberts P, Palmer AJ, et al. The Urgent Need for Evidence in Arthroscopic Meniscal Surgery: A Systematic Review of the Evidence for Operative Management of Meniscal Tears. Am J Sports Med 2016.

7. Yim JH, Seon JK, Song EK, et al. A comparative study of meniscectomy and nonoperative treatment for degenerative horizontal tears of the medial meniscus. Am J Sports Med 2013:41:1565-70.

8. Sihvonen R, Paavola M, Malmivaara A, et al. Arthroscopic partial meniscectomy versus sham surgery for a degenerative meniscal tear. N Engl J Med 2013;369:2515-24.

9. Kise NJ, Risberg MA, Stensrud S, et al. Exercise therapy versus arthroscopic partial menisectomy; Effect on mechanical symptoms. The 13th Scandinavian Congress of Medicine and Science in Sports. Norefjell, Norway 2016.

10. Lattermann C, Gomoll AH, Cole BJ. Arthroscopic partial meniscectomy for degenerative meniscal tear. $N$ Engl $J$ Med 2014;370:1260

11. Kim S, Bosque J, Meehan JP, et al. Increase in outpatient knee arthroscopy in the United States: a comparison of National Surveys of Ambulatory Surgery, 1996 and 2006. J Bone Joint Surg Am 2011;93:994-1000.

12. Holmes R, Moschetti W, Martin B, et al. Effect of evidence and changes in reimbursement on the rate of arthroscopy for osteoarthritis. Am J Sports Med 2013;41:1039-43.

13. Moseley JB, O'Malley K, Petersen NJ, et al. A controlled trial of arthroscopic surgery for osteoarthritis of the knee. N Engl J Med 2002;347:81-8

14. Zou K, Wong J, Abdullah N, et al. Examination of overall treatment effect and the proportion attributable to contextual effect in osteoarthritis: meta-analysis of randomised controlled trials. Ann Rheum Dis 2016;75:1964-70.

15. Wartolowska K, Judge A, Hopewell S, et al. Use of placebo controls in the evaluation of surgery: systematic review. BMJ 2014;348:g3253.

16. Jonas WB, Crawford C, Colloca L, et al. To what extent are surgery and invasive procedures effective beyond a placebo response? A systematic review with meta-analysis of randomised, sham controlled trials. BMJ Open 2015;5:e009655.

17. Hare KB, Lohmander LS, Christensen R, et al. Arthroscopic partial meniscectomy in middle-aged patients with mild or no knee osteoarthritis: a protocol for a double-blind, randomized shamcontrolled multi-centre trial. BMC Musculoskelet Disord 2013;14:71.

18. Roos EM, Roos HP, Ekdahl C, et al. Knee injury and Osteoarthritis Outcome Score (KOOS)-validation of a Swedish version. Scand $J$ Med Sci Sports 1998;8:439-48.

19. Collins NJ, Prinsen CA, Christensen R, et al. Knee Injury and Osteoarthritis Outcome Score (KOOS): systematic review and meta-analysis of measurement properties. Osteoarthritis Cartilage 2016;24:1317-29.

20. Kroman SL, Roos EM, Bennell KL, et al. Measurement properties of performance-based outcome measures to assess physical function in young and middle-aged people known to be at high risk of hip and/or knee osteoarthritis: a systematic review. Osteoarthritis Cartilage 2014;22:26-39.

21. Bremander AB, Dahl LL, Roos EM. Validity and reliability of functional performance tests in meniscectomized patients with or without knee osteoarthritis. Scand J Med Sci Sports 2007;17:120-7.

22. Ranstam J, Turkiewicz A, Boonen S, et al. Alternative analyses for handling incomplete follow-up in the intention-to-treat analysis: the randomized controlled trial of balloon kyphoplasty versus nonsurgical care for vertebral compression fracture (FREE). BMC Med Res Methodol 2012;12:35.

23. Hare KB, Lohmander LS, Roos EM. The challenge of recruiting patients into a placebo-controlled surgical trial. Trials 2014;15:167.

24. Feys F, Bekkering GE, Singh K, et al. Do randomized clinical trials with inadequate blinding report enhanced placebo effects for intervention groups and nocebo effects for placebo groups? Syst Rev 2014;3:14 